\section{Fatores associados à interrupção do aleitamento materno exclusivo de lactentes nascidos com baixo peso assistidos na atenção básica}

\author{
Factors associated with interruption of exclusive \\ breastfeeding in low birth weight infants \\ receiving primary care
}

\author{
Maria Teresa Cera Sanches 1 \\ Gabriela dos Santos Buccini 1 \\ Suely Godoy Agostinho Gimeno 2 \\ Tereza Etsuko da Costa Rosa 1 \\ Andrea Wander Bonamigo ${ }^{3}$
}

1 Instituto de Saúde, Secretaria Estadual de Saúde de São Paulo, São Paulo, Brasil.

2 Departamento de Medicina Preventiva, Universidade Federal de São Paulo, São Paulo, Brasil.

3 Supervisão Técnica de Saúde de Pirituba/Perus, Secretaria Municipal de Saúde de São Paulo, São Paulo, Brasil.

Correspondência M. T. C. Sanches Instituto de Saúde, Secretaria Estadual de Saúde de São Paulo.

Rua Santo Antonio 590, 3o andar, São Paulo, SP 013140-000, Brasil. mtsanches@isaude.sp.gov.br

\section{Abstract}

This study aimed to identify factors associated with interruption of exclusive breastfeeding (EBF) in low birth weight infants receiving primary care. This was a cross-sectional study of 170 infants treated at primary care units on the urban periphery of São Paulo, Brazil. The sample included infants with birth weight $\leq 2,500 \mathrm{~g}$ (including twins) and 5-minute Apgar $\geq 7$, followed until the third month of life, and excluded infants/mothers with complications that would impede EBF, besides infant/maternal deaths. Data were collected from forms completed during consultations and patient records. Prevalence ratios were obtained by Poisson regression. The following factors were associated with interruption of EBF in the first three months of life: maternal age $\leq 18$ years; informal employment (protective factor); alcohol intake during pregnancy; < 6 prenatal visits; $m u l$ tiple gestations; birth weight $\leq 2,000$; difficulty breastfeeding in the first month; complaints in breastfeeding during the first month; and use of pacifiers in the first two months. Prior knowledge of these factors can help plan measures and policies to increase EBF rates among low birth weight infants.

Low Birth Weight Infant; Breast Feeding; Primary Health care

\section{Introdução}

O aleitamento materno é uma prática que contribui para a redução da morbimortalidade, especialmente por seus diversos benefícios físicos e mentais, entre os recém-nascidos de baixo peso e prematuros 1,2,3,4. Dentre as principais vantagens do uso do leite humano nessa população estão a prevenção de infecções, auxílio na maturação gastrintestinal e proteção da retinopatia da prematuridade. Além disso, no desenvolvimento a médio e longo prazo, a amamentação apresenta até vantagens no desempenho cognitivo e na prevenção de problemas metabólicos e, consequentemente, na de doenças cardiovasculares na vida adulta 2,4. Não obstante, a literatura indica uma prevalência muito baixa do aleitamento materno exclusivo (AME) em bebês no geral e menor ainda nos recém-nascidos de baixo peso 2,3,4.

No Brasil, contextualizando-se a elevação das taxas de aleitamento materno na assistência perinatal, a partir de 2000, o Ministério da Saúde implantou o Programa de Humanização no Prénatal e Nascimento ${ }^{5}$. Esse propõe a humanização e qualificação da assistência como estratégia para a melhoria do acesso, da cobertura e da qualidade do pré-natal, parto e puerpério. Como parte desse Programa, aprova também a Norma de Atenção Humanizada ao Recém-nascido de Baixo Peso (Método Canguru) 4.

Estudos têm apontado o Método Canguru, sistematizando rotinas hospitalares mais huma- 
nizadas, com uma equipe adequadamente capacitada para o manejo do aleitamento materno e com o apoio do Banco de Leite Humano, como ótima estratégia para a promoção de maior duração do aleitamento materno nos recém-nascidos de baixo peso 3,6,7,8,9,10. Entretanto, apesar da própria estratégia assinalar a importância da referência dos recém-nascidos de baixo peso às unidades básicas de saúde (UBS) após a alta hospitalar, esta ainda não prevê formas de integração entre ambos os serviços.

Em relação a uma abordagem sistematizada do aleitamento materno na Atenção Básica, o Ministério da Saúde iniciou a implantação da Rede Amamenta Brasil a partir de 2008 11. Essa estratégia, que se apoia no princípio da educação permanente em saúde, propõe uma revisão da atuação da equipe interdisciplinar na amamentação e uma mudança na visão desta prática entre os profissionais da Atenção Básica. Pressupõe capacitação dos profissionais de saúde, baseada no aconselhamento em amamentação 12, levando-se em consideração características socioculturais da mulher. Propõe, também, respeitando-se as especificidades locais e regionais, a pactuação coletiva das ações prioritárias para a amamentação, desde o pré-natal até o seguimento ambulatorial.

Percebe-se que as políticas e os programas para a promoção do aleitamento materno têm se apoiado na literatura especializada no sentido de incluir nas ações assistenciais prestadas às mulheres aspectos relacionados com o apoio psicológico e os socioculturais 12,13,14,15,16, que podem interferir negativamente na duração da amamentação, principalmente para os recémnascidos de baixo peso 4,7,17,18,19.

Sabe-se, no entanto, pela mesma literatura, que os determinantes que influenciam o sucesso do aleitamento materno são inúmeros e de abordagem bastante complexa 14,20. Dessa forma, ampliar os conhecimentos sobre os fatores que interferem na amamentação, em especial entre os nascidos de baixo peso atendidos na Atenção Básica, pode aperfeiçoar os subsídios para as políticas públicas nesse âmbito.

O presente estudo teve como objetivo identificar os fatores associados à interrupção da amamentação exclusiva dos lactentes nascidos de baixo peso assistidos na Atenção Básica.

\section{Métodos}

\section{População e território de estudo}

Trata-se de um estudo transversal com lactentes nascidos de baixo peso que, no terceiro mês de vida, permaneciam (ou não) em AME. Para tal, utiliza-se dados de uma coorte de lactentes que compareceram, a partir da demanda espontânea, às UBS selecionadas para consulta de puerpério e que atenderam aos seguintes critérios de inclusão: nascidos no período de janeiro de 2007 a março de 2008, peso de nascimento abaixo de 2.500g, Apgar (5') igual ou maior que 7 e que realizaram o seguimento ambulatorial até o terceiro mês. Os bebês nascidos de partos gemelares, dentro desses critérios, também foram incluídos. Excluíram-se os casos que apresentaram malformações, síndromes genéticas, meningite, broncodisplasia pulmonar, hemorragia intraventricular (graus III e IV) que pudessem impedir totalmente a amamentação e alterações psíquicas (das mães), além de casos de óbito do bebê ou da mãe.

As UBS selecionadas pertenciam à Supervisão Técnica de Saúde de Pirituba/Perus (STSP/P), área situada na região periférica norte do Município de São Paulo. O estudo abrangeu 23 das 24 UBS existentes na época do estudo, sendo 9 UBS tradicionais, 11 com a Estratégia Saúde da Família (ESF) e 3 unidades mistas (tradicionais e ESF). A UBS (tradicional) que não participou do estudo alegou dificuldades administrativas e gerenciais no período da coleta de dados.

\section{Variáveis do estudo}

A variável desfecho do estudo foi a interrupção do AME até o terceiro mês. Considerou-se em AME, segundo a classificação sugerida pela Organização Mundial da Saúde (OMS) 21, aquelas que recebiam leite materno exclusivo, ofertado diretamente da mama ou ordenhado e oferecido, e nenhum outro líquido ou sólido, com exceção de gotas ou xaropes de vitaminas, minerais ou medicamentos.

As variáveis independentes do estudo, definidas com base na literatura científica da área e nas condições de coleta das informações proporcionadas pelo tipo de estudo proposto, estão apresentadas na Figura 1. Essas foram agrupadas em blocos, segundo características: sociodemográficas e de estilo de vida das mães (Bloco 1), gestacionais e do parto (Bloco 2), condições de nascimento dos lactentes (Bloco 3), evolução clínica e nutrição dos lactentes na fase hospitalar (Bloco 4), prática inicial na amamentação (Bloco 5), seguimento ambulatorial nas unidades de saúde no primeiro (Bloco 6) e no segundo meses (Bloco 7). 


\section{Figura 1}

Referencial teórico para investigação dos fatores associados à interrupção do aleitamento materno exclusivo de lactentes nascidos com baixo peso assistidos na Atenção Básica. Supervisão Técnica de Saúde de Pirituba/Perus, São Paulo, Brasil, 2007.

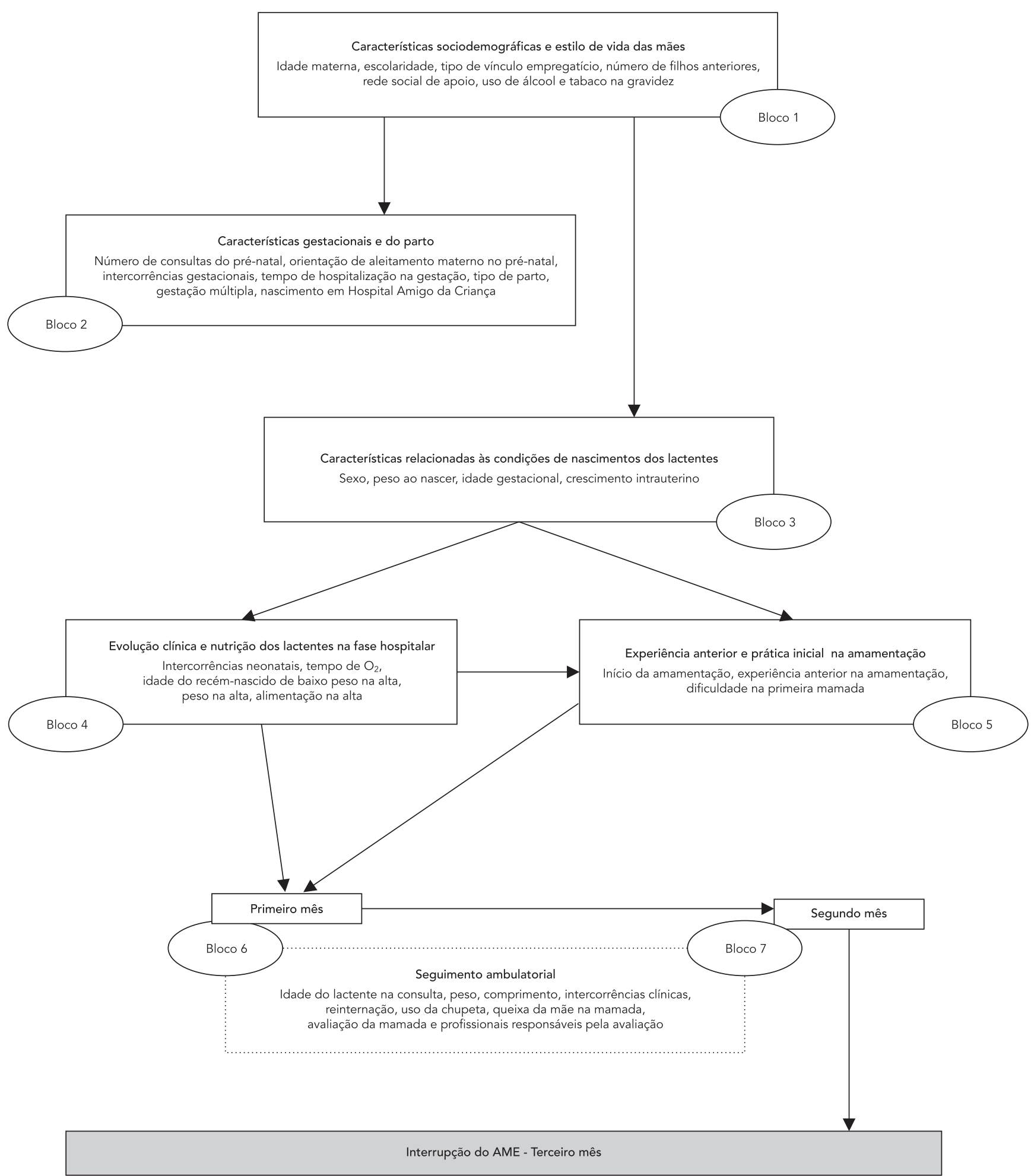




\section{Coleta de dados}

A coleta de dados se deu na medida em que as mães, cujos filhos haviam nascido no período de interesse, compareciam às UBS para as consultas de rotina de puerpério, ou recebiam visitas domiciliares dos profissionais da ESF, com retornos mensais até o terceiro mês.

As informações foram coletadas por meio de dois instrumentos especialmente elaborados e pré-testados para este estudo:

1) Formulário 1 - Dados gestacionais e do nascimento: referente aos dados gestacionais e condições de nascimento dos lactentes com baixo peso ao nascer e de identificação das mães, coletados pelos próprios pesquisadores diretamente dos prontuários dos lactentes nas UBS. Essa coleta foi executada por seis auxiliares de pesquisa de nível superior, previamente treinados;

2) Formulário 2 - Seguimento ambulatorial dos lactentes com baixo peso ao nascer: referente aos dados clínicos após a alta hospitalar: tempo de duração do aleitamento materno exclusivo e introdução de outros alimentos, uso de chupeta, dificuldades na prática da amamentação e dados de avaliação da mamada. Outros dados gestacionais considerados importantes para o estudo e que não constavam no prontuário (Formulário 1), tais como orientação sobre aleitamento materno no pré-natal, uso de álcool e tabaco durante a gestação e condições sobre o início do aleitamento materno foram coletados neste formulário. Esses foram preenchidos pelos profissionais de saúde, cujo treinamento está detalhado a seguir, durante as consultas nas UBS, ou pelos pesquisadores, após as consultas. Ressalta-se que, para evitar o viés do informante, o questionamento sobre a avaliação da mamada foi realizado pelos pesquisadores, em geral, por meio de entrevista, após a consulta, ou por meio telefônico no domicílio da mãe.

Para garantir a adesão das UBS ao estudo realizaram-se inicialmente Oficinas de Sensibilização envolvendo todos os gerentes das unidades, além dos responsáveis pela saúde da criança. Realizaram-se também Oficinas de Capacitação com médicos generalistas, pediatras e enfermeiros de todas as UBS do estudo para garantia da correta utilização do Formulário 2. Outro cuidado despendido foi a confecção de instrumento em cores distintas com a finalidade de facilitar a sua visualização nos prontuários e agilizar a coleta.

Adotaram-se duas estratégias em todo o período de coleta de dados, assegurando-se a confiabilidade e qualidade dos dados: (a) supervisão de campo semanal em todas as UBS para acompanhamento da coleta de dados com os profis- sionais de saúde; (b) contato com as mães por meio telefônico ou visitas domiciliares, a fim de complementar as informações.

\section{Análise estatística}

Para avaliação conjunta dos fatores associados à interrupção do AME e as variáveis agrupadas em blocos, utilizou-se a análise de regressão de Poisson múltipla hierarquizada. A ordem estabelecida para os blocos variou de acordo com a influência direta ou indireta com que atuariam sobre a interrupção do AME no terceiro mês, de acordo com o modelo teórico estabelecido (Figura 1).

As análises iniciaram-se com a verificação da existência de associação entre cada variável candidata a compor o modelo hierarquizado e o desfecho. Adotou-se o nível de significância $\leq$ 0,10 (qui-quadrado de Pearson) para selecionar os fatores para as análises de regressão múltipla subsequentes.

Em seguida, realizou-se análise múltipla dentro de cada bloco e foram mantidos para as próximas análises os fatores que apresentaram nível de significância $\leq 0,10$. Após essa análise interna de cada um dos blocos, realizou-se a modelagem hierarquizada entre os blocos, considerando-se para interpretação do resultado final associação estatisticamente significativa $(p \leq 0,05)$ entre um determinado fator e a interrupção do AME, após o ajuste para os fatores do mesmo bloco e dos fatores dos blocos hierárquicos superiores.

Para criação do banco de dados utilizou-se o programa Epi Info versão 2000 (Centers for Disease Control and Prevention, Atlanta, Estados Unidos), com dupla digitação, e para a análise estatística o programa Stata versão 8.2 (Stata Corp., College Station, Estados Unidos).

\section{Cuidados éticos}

A concordância para participar da pesquisa foi feita mediante assinatura das mães do termo de consentimento livre e esclarecido. O projeto de pesquisa foi aprovado pelo Comitê de Ética em Pesquisa (nº. 0209/2006) da Secretaria Municipal de Saúde de São Paulo e também pelo Comitê de Ética do Instituto de Saúde (nº. 013/2006) e está de acordo com a Resolução $n^{\circ} .196 / 96$ do Conselho Nacional de Saúde.

\section{Resultados}

No período do estudo procuraram as UBS participantes do projeto 246 bebês nascidos com 
baixo peso, dos quais 199 atenderam aos critérios de inclusão (elegíveis). No decorrer da coleta de dados ocorreram algumas perdas pelos seguintes motivos: mudanças de endereço ( $\mathrm{n}=$ 16); perda do prontuário ou ficha dos lactentes, ocorrida na UBS $(n=4)$; e óbito da mãe $(n=1)$. Algumas mães se recusaram a participar ( $\mathrm{n}=$ 8), totalizando 29 crianças $(14,6 \%)$ que não participaram do estudo. Participantes e não participantes foram comparados segundo idade, escolaridade e trabalho da mãe, idade gestacional, gestação múltipla, pré-natal, sexo do bebê e peso ao nascer (informações que constam da declaração de nascidos vivos). Nas análises dessas variáveis não foram constatadas diferenças estatisticamente significativas entre os dois grupos ( $\mathrm{p}>0,05)$. Os resultados deste estudo referiram-se, portanto, a 170 duplas de mães/ lactentes nascidos com baixo peso $(85,4 \%$ do total de recém-nascidos elegíveis), que mantiveram o seguimento ambulatorial, no mínimo, até o terceiro mês (Figura 2).

A média de idade das mães foi de 24 anos [desvio padrão (DP) $=6,30$ anos] e de escolaridade 8 anos (DP = 2,71 anos). A maioria (73\%) referiu trabalhar de modo informal ou não trabalhar fora do ambiente doméstico e 10\% relataram consumir bebida alcoólica diariamente durante a gestação.
Do total de lactentes, $69 \%$ nasceram em hospitais credenciados como "Amigo da Criança", que utilizavam o Método Canguru como rotina na assistência. Observou-se maior proporção (55\%) do sexo feminino, a média de idade gestacional foi de 35 semanas, o peso médio de nascimento foi de $2.165 \mathrm{~g}$ (DP $=293,58 \mathrm{~g}$ ) e $63 \%$ eram adequados para a idade gestacional.

Quanto à prática da amamentação, identificou-se 58,5\% de lactentes em AME no primeiro, $43,4 \%$ no segundo e 33,3\% no terceiro mês.

A distribuição dos lactentes nascidos de baixo peso segundo a situação do AME no terceiro mês e as variáveis de interesse selecionadas para a entrada no modelo $(\mathrm{p} \leq 0,10)$ estão apresentadas na Tabela 1. Na análise de regressão univariada as seguintes variáveis foram selecionadas por mostrarem associação estatisticamente significativa ( $\mathrm{p} \leq 0,05)$ : idade da mãe; vínculo empregatício; uso de bebida alcoólica na gestação (Bloco 1); número de consultas de pré-natal; tipo de gestação (Bloco 2); peso ao nascer (Bloco 3); dificuldade nas primeiras mamadas (Bloco 5); queixa da mãe na mamada no primeiro mês; uso de chupeta no primeiro mês (Bloco 6); queixa da mãe na mamada no segundo mês; e uso de chupeta no segundo mês (Bloco 7). Além dessas, foram selecionadas como controle por apresentarem níveis de significância iguais ou inferiores a 10\%: idade

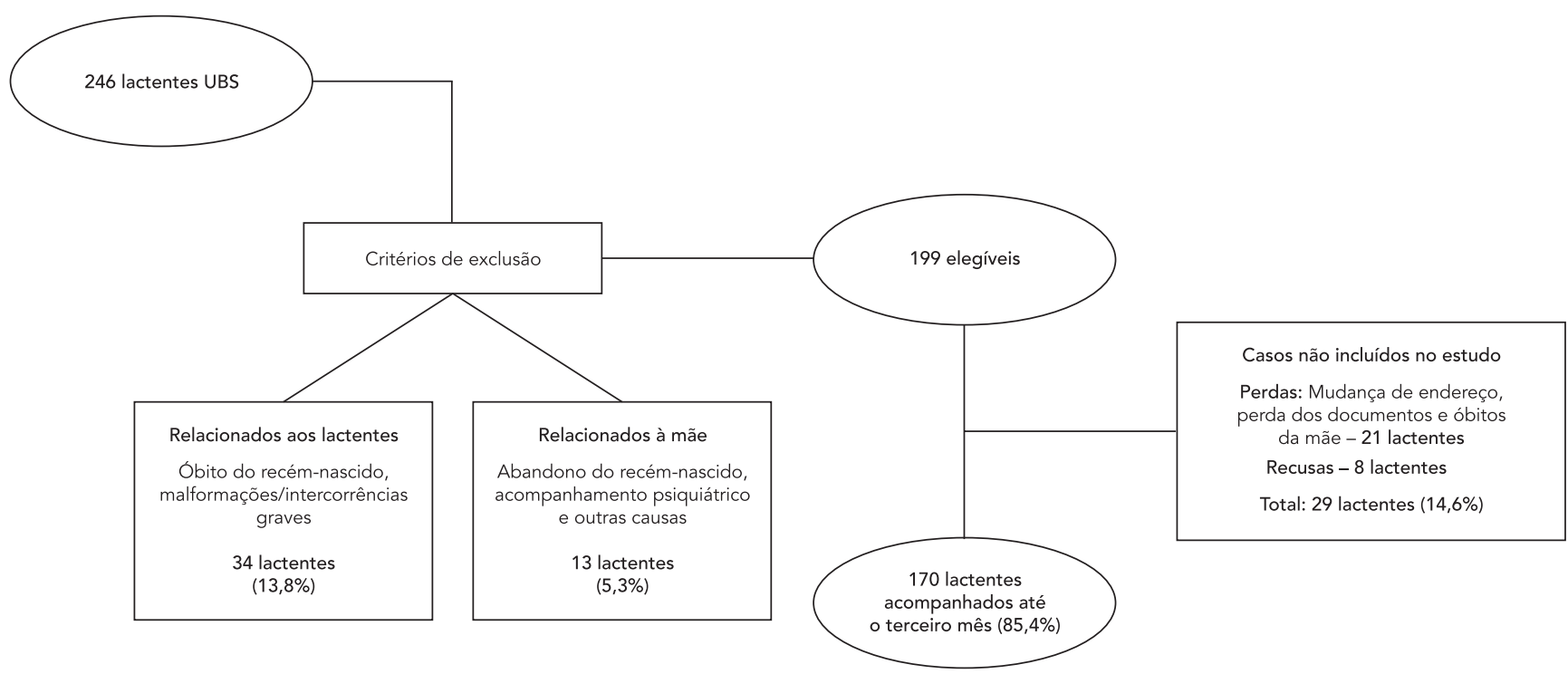


Distribuição dos lactentes nascidos com baixo peso segundo a situação do aleitamento materno exclusivo (AME) no terceiro mês e razões de prevalência (RP) e valores de p da análise univariada de Poisson. Supervisão Técnica de Saúde de Pirituba/ Perus, São Paulo, Brasil, 2007.

\begin{tabular}{|c|c|c|c|c|c|c|c|c|}
\hline \multirow[t]{2}{*}{ Bloco/Variável } & \multicolumn{2}{|c|}{ AME } & \multicolumn{2}{|c|}{ Não AME } & \multicolumn{2}{|c|}{ Total } & \multirow[t]{2}{*}{$\mathrm{RP}$} & \multirow{2}{*}{$\begin{array}{l}\text { Valor } \\
\text { de } p\end{array}$} \\
\hline & $\mathrm{n}$ & $\%$ & $\mathrm{n}$ & $\%$ & $\mathrm{n}$ & $\%$ & & \\
\hline \multicolumn{9}{|c|}{ Bloco 1 - Características sociodemográficas e } \\
\hline \multicolumn{9}{|c|}{ de estilo de vida das mães } \\
\hline \multicolumn{9}{|c|}{ Idade da mãe (anos) } \\
\hline$<18$ & 2 & 11 & 16 & 89 & 18 & 100 & 1,41 & 0,001 \\
\hline $35-42$ & 4 & 33 & 8 & 67 & 12 & 100 & 1,06 & 0,802 \\
\hline $18-34$ & 49 & 37 & 84 & 63 & 133 & 100 & 1,00 & \\
\hline \multicolumn{9}{|c|}{ Escolaridade da mãe (anos) } \\
\hline $0-4$ & 6 & 38 & 10 & 62 & 16 & 100 & 0,96 & 0,850 \\
\hline $5-8$ & 15 & 23 & 50 & 77 & 65 & 100 & 1,23 & 0,313 \\
\hline 9 ou mais & 32 & 40 & 48 & 60 & 80 & 100 & 1,00 & \\
\hline \multicolumn{9}{|c|}{ Vínculo empregatício } \\
\hline Informal & 45 & 38 & 75 & 62 & 120 & 100 & 0,80 & 0,033 \\
\hline Formal & 9 & 21 & 33 & 79 & 42 & 100 & 1,00 & \\
\hline \multicolumn{9}{|l|}{ Uso de álcool } \\
\hline Sim & 2 & 13 & 13 & 87 & 15 & 100 & 1,35 & 0.011 \\
\hline Não & 53 & 36 & 95 & 64 & 148 & 100 & 1,00 & \\
\hline \multicolumn{9}{|c|}{ Bloco 2 - Características da gestação e parto } \\
\hline \multicolumn{9}{|c|}{ Pré-natal (consultas) } \\
\hline$<6$ & 9 & 16 & 47 & 84 & 56 & 100 & 1,48 & $<0,001$ \\
\hline$\geq 6$ & 46 & 43 & 60 & 57 & 106 & 100 & 1,00 & \\
\hline \multicolumn{9}{|c|}{ Gestação múltipla } \\
\hline $\operatorname{Sim}$ & 3 & 12 & 22 & 88 & 25 & 100 & 1,41 & 0,001 \\
\hline Não & 52 & 38 & 86 & 62 & 138 & 100 & 1,00 & \\
\hline \multicolumn{9}{|c|}{ Bloco 3 - Características do nascimento } \\
\hline \multicolumn{9}{|c|}{ Peso ao nascimento $(\mathrm{g})$} \\
\hline$\leq 2.000$ & 4 & 12 & 29 & 88 & 33 & 100 & 1,45 & $<0,001$ \\
\hline$>2.000$ & 51 & 39 & 79 & 61 & 130 & 100 & 1,00 & \\
\hline \multicolumn{9}{|c|}{ Idade gestacional (semanas) } \\
\hline$\leq 36$ & 30 & 41 & 44 & 59 & 74 & 100 & 0,83 & 0,104 \\
\hline$>36$ & 25 & 28 & 64 & 72 & 89 & 100 & 1,00 & \\
\hline \multicolumn{9}{|c|}{ Bloco 4 - Características da fase hospitalar } \\
\hline \multicolumn{9}{|c|}{ Tempo de uso de $\mathrm{O}_{2}$ (dias) } \\
\hline$>5$ & 2 & 12 & 15 & 88 & 17 & 100 & 1,34 & 0,010 \\
\hline $2-5$ & 19 & 40 & 28 & 60 & 47 & 100 & 0,91 & 0,490 \\
\hline$\leq 1$ & 34 & 34 & 65 & 66 & 99 & 100 & 1,00 & \\
\hline \multicolumn{9}{|c|}{ Bloco 5 - Prática inicial da amamentação } \\
\hline \multicolumn{9}{|c|}{ Dificuldade na primeira mamada } \\
\hline Sim & 22 & 24 & 69 & 76 & 91 & 100 & 1,40 & 0,007 \\
\hline Não & 33 & 46 & 39 & 54 & 72 & 100 & 1,00 & \\
\hline
\end{tabular}


Tabela 1 (continuação)

\begin{tabular}{|c|c|c|c|c|c|c|c|c|}
\hline \multirow[t]{2}{*}{ Bloco/Variável } & \multicolumn{2}{|c|}{ AME } & \multicolumn{2}{|c|}{ Não AME } & \multicolumn{2}{|c|}{ Total } & \multirow[t]{2}{*}{ RP } & \multirow{2}{*}{$\begin{array}{l}\text { Valor } \\
\text { de } p\end{array}$} \\
\hline & n & $\%$ & $\mathrm{n}$ & $\%$ & $\mathrm{n}$ & $\%$ & & \\
\hline \multicolumn{9}{|c|}{ Bloco 6 - Características no primeiro mês de } \\
\hline \multicolumn{9}{|c|}{ vida } \\
\hline \multicolumn{9}{|c|}{ Reinternação até o primeiro mês } \\
\hline Sim & 5 & 63 & 3 & 37 & 8 & 100 & 0,56 & 0,205 \\
\hline Não & 49 & 33 & 101 & 67 & 150 & 100 & 1,00 & \\
\hline \multicolumn{9}{|c|}{ Queixa da mãe na mamada } \\
\hline Sim & 25 & 27 & 69 & 73 & 94 & 100 & 1,80 & 0,001 \\
\hline Não & 29 & 59 & 20 & 41 & 49 & 100 & 1,00 & \\
\hline \multicolumn{9}{|c|}{ Uso de chupeta no primeiro mês } \\
\hline Sim & 6 & 13 & 42 & 87 & 48 & 100 & 1,54 & $<0,001$ \\
\hline Não & 48 & 43 & 63 & 57 & 111 & 100 & 1,00 & \\
\hline \multirow{2}{*}{\multicolumn{9}{|c|}{$\begin{array}{l}\text { Bloco } 7 \text { - Características no segundo mês de } \\
\text { vida }\end{array}$}} \\
\hline & & & & & & & & \\
\hline \multicolumn{9}{|c|}{ Queixa da mãe na mamada } \\
\hline Sim & 19 & 23 & 62 & 77 & 81 & 100 & 1,93 & $<0,001$ \\
\hline Não & 35 & 60 & 23 & 40 & 58 & 100 & 1,00 & \\
\hline \multicolumn{9}{|c|}{ Uso de chupeta no segundo mês } \\
\hline $\operatorname{Sim}$ & 10 & 14 & 64 & 86 & 74 & 100 & 1,73 & $<0,001$ \\
\hline Não & 44 & 50 & 44 & 50 & 88 & 100 & 1,00 & \\
\hline \multicolumn{9}{|c|}{ Avaliação da mamada } \\
\hline Não realizada & 17 & 30 & 40 & 70 & 57 & 100 & 1,24 & 0,098 \\
\hline Realizada & 36 & 43 & 47 & 57 & 83 & 100 & 1,00 & \\
\hline
\end{tabular}

gestacional (Bloco 3); tempo de uso de oxigênio (Bloco 4) e avaliação da mamada (Bloco 7).

As variáveis número de filhos anteriores, rede social de apoio e uso de tabaco (Bloco 1); orientação sobre aleitamento materno no prénatal, intercorrências da mãe durante a gestação, tempo de hospitalização na gestação, tipo de parto e nascimento em Hospital Amigo da Criança (Bloco 2); sexo e crescimento intrauterino (Bloco 3); intercorrências neonatais, idade, peso e alimentação na alta hospitalar (Bloco 4); início da amamentação e experiência anterior na amamentação (Bloco 5); idade, peso e comprimento do lactente nas consultas, intercorrências clínicas e profissional responsável pela avaliação da mamada (Blocos 6 e 7) não mostraram associação em um nível de significância que justificasse suas seleções para entrada nos modelos.

A Tabela 2 apresenta as variáveis selecionadas para os modelos e que permaneceram após ajuste dentro de cada bloco e o ajuste, subsequente, entre os blocos. As variáveis excluídas quando ajustadas com as demais foram: escolaridade da mãe (Bloco 1); idade gestacional (Bloco 3); tempo de uso de oxigênio (Bloco 4); e reinternação (Bloco 6).
Como resultado da análise de regressão de Poisson hierarquizada, verificou-se que os fatores associados à interrupção do aleitamento materno exclusivo no terceiro mês dos lactentes nascidos de baixo peso da região de Pirirtuba/ Perus foram: idade da mãe (< 18 anos), vínculo empregatício do tipo informal (como fator de proteção), mãe ter ingerido álcool diariamente na gestação, mãe ter realizado menos de seis consultas de pré-natal, gestação múltipla, peso de nascimento igual ou inferior a $2.000 \mathrm{~g}$, dificuldade do bebê nas primeiras mamadas, presença de queixa da mãe na amamentação no primeiro mês e uso de chupeta no primeiro e segundo meses. Vale salientar que a variável idade foi categorizada em três níveis (< 18 anos, 35 a 42 e 18 a 34, esta como categoria de referência), sendo que a associação significativa foi observada somente para a faixa etária mais jovem.

Com exceção de número de consultas de pré-natal, queixa da mãe na mamada no primeiro mês e uso de chupeta no segundo mês, que apresentaram valores de p próximos a 0,05, os valores de $\mathrm{p}$ menores que 0,01 das demais variáveis indicam forte associação destes fatores com a interrupção do AME, embora os valores das razões de prevalência, adjacentes ao valor 1 
Valores das razões de prevalências (RP), por ponto e por intervalo de $95 \%$ de confiança (IC95\%), para fatores associados à interrupção do aleitamento materno exclusivo no terceiro mês. Modelos de regressão de Poisson múltipla hierarquizada. Supervisão Técnica de Saúde de Pirituba/Perus, São Paulo, Brasil, 2007.

\begin{tabular}{|c|c|c|}
\hline Variáveis & RP [IC95\%] & Valor de $\mathrm{p}$ \\
\hline \multicolumn{3}{|c|}{ Modelo 1 - Características sociodemográficas e de estilo devida mães } \\
\hline \multicolumn{3}{|c|}{ (Bloco 1) } \\
\hline \multicolumn{3}{|c|}{ Idade da mãe (anos) } \\
\hline$<18$ & $1,59[1,24-2,02]$ & 0,00 \\
\hline $35-42$ & $0,97[0,66-1,43]$ & 0,87 \\
\hline $18-34$ & 1,00 & \\
\hline \multicolumn{3}{|c|}{ Vínculo empregatício } \\
\hline Informal & $0,70[0,55-0,89]$ & 0,003 \\
\hline Formal & 1,00 & \\
\hline \multicolumn{3}{|l|}{ Uso de álcool } \\
\hline Sim & $1,50[1,16-1,93]$ & 0,002 \\
\hline Não & 1,00 & \\
\hline \multicolumn{3}{|c|}{ Modelo 2 - Características da gestação e parto (Bloco 2) * } \\
\hline \multicolumn{3}{|c|}{ Pré-natal (consultas) } \\
\hline$<6$ & $1,30[1,06-1,60]$ & 0,013 \\
\hline$\geq 6$ & 1,00 & \\
\hline \multicolumn{3}{|l|}{ Gestação múltipla } \\
\hline Sim & $1,50[1,21-1,85]$ & 0,000 \\
\hline Não & 1,00 & \\
\hline \multicolumn{3}{|c|}{ Modelo 3 - Características do nascimento (Bloco 3) ** } \\
\hline \multicolumn{3}{|c|}{ Peso ao nascimento $(\mathrm{g})$} \\
\hline$\leq 2.000$ & $1,37[1,12-1,69]$ & 0,002 \\
\hline$>2.000$ & 1,00 & \\
\hline \multicolumn{3}{|c|}{ 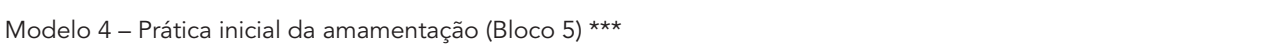 } \\
\hline \multicolumn{3}{|c|}{ Dificuldade na primeira mamada } \\
\hline $\operatorname{Sim}$ & $1,44[1,14-1,82]$ & 0,003 \\
\hline Não & 1,00 & \\
\hline \multicolumn{3}{|c|}{ Modelo 5 - Características no primeiro mês de vida (Bloco 6) \# } \\
\hline \multicolumn{3}{|c|}{ Queixa da mãe na mamada } \\
\hline Sim & $1,48[1,05-2,10]$ & 0,024 \\
\hline Não & 1,00 & \\
\hline \multicolumn{3}{|c|}{ Uso de chupeta no primeiro mês } \\
\hline Sim & $1,42[1,15-1,76]$ & 0,001 \\
\hline Não & 1,00 & \\
\hline \multicolumn{3}{|c|}{ Modelo 6 - Características no segundo mês de vida (Bloco 7) \#\# } \\
\hline \multicolumn{3}{|c|}{ Uso de chupeta no segundo mês } \\
\hline Sim & $1,53[1,10-2,13]$ & 0,013 \\
\hline Não & 1,00 & \\
\hline \multicolumn{3}{|c|}{ Avaliação da mamada } \\
\hline Não realizada & $1,27[0,98-1,65]$ & 0,070 \\
\hline Realizada & 1,00 & \\
\hline
\end{tabular}

* Ajustado pelo Modelo 1;

** Ajustado pelos Modelos 1 e 2;

*** Ajustado pelos Modelos 1, 2 e 3;

\# Ajustado pelos Modelos 1, 2, 3 e 5;

\#\# Ajustado pelos Modelos 1, 2, 3, 5 e 6 . 
de todas as variáveis, indiquem magnitudes não muito elevadas.

\section{Discussão}

Pelos resultados apresentados verificamos que as características e estilo de vida da mãe (ter menos que 18 anos, trabalhar sem vínculo formal e ter ingerido bebida alcoólica durante a gestação), quando ajustados entre si e entre os blocos, mantiveram a força e a magnitude de associação, indicando a sua relevância na determinação da interrupção do AME dos lactentes nascidos com baixo peso, de forma independente em relação às demais variáveis. Entre as características gestacionais e do parto, bem como as relacionadas às condições de nascimento, verificou-se que a probabilidade de ocorrência de interrupção precoce do AME é consistentemente maior entre as mulheres que realizaram um número de consultas de pré-natal inferior a seis, entre gêmeos, entre os nascidos com peso igual ou inferior a $2.000 \mathrm{~g}$ e entre as mães que tiveram dificuldades nas primeiras mamadas, independentemente da idade, do vínculo de trabalho e do uso de bebida alcoólica na gestação. Dos fatores relacionados às condições após a alta hospitalar investigados, mostraram associação com a interrupção do AME no terceiro mês a presença de queixa da mãe na amamentação no primeiro mês e o uso de chupeta, apresentados tanto no primeiro quanto no segundo mês de vida do recém-nascido, independentemente dos fatores antes mencionados.

No que diz respeito à idade da mãe, ser jovem tem sido apontado como um dos fatores de risco à continuidade do $\mathrm{AME}$ 20,22, o que é consistente com o resultado do presente estudo. Exame detalhado dos resultados (não mostrados em tabelas) das análises de regressão hierarquizada mostra que, mesmo após todos os ajustes realizados, a categoria mencionada manteve a magnitude e a significância estatística, indicando que, entre as mães mais jovens, a probabilidade de interromper o AME é maior, independentemente de todas as demais condições, comparando-se àquelas do grupo etário entre 18 e 34 anos.

No entanto, outros estudos, com dados hospitalares, empreendidos dentro do contexto da Iniciativa Hospital Amigo da Criança, do Banco de Leite Humano e do Método Canguru não evidenciaram a faixa etária mais jovem como fator de risco para a interrupção precoce do $\mathrm{AME}$ 7,18. Uma possível explicação para a contradição entre os estudos pode ter relação com as práticas humanizadas adotadas nesses locais, que os distinguem dos demais e que podem promover a superação dos obstáculos ao AME, até mesmo os das mães mais jovens com seus lactentes nascidos de baixo peso.

A ingestão de bebida alcoólica pela mãe é apontada como comportamento associado à redução do tempo de amamentação ${ }^{22}$, resultado também observado neste estudo. Embora a presente pesquisa tenha focado sobre o comportamento durante a gestação, não é difícil generalizar a sua ocorrência para o período posterior ao parto, durante a amamentação. Se isso for verdade, o achado é congruente com o estudo que identificou uma importante correlação inversa entre os hábitos de consumo alcoólico pela mãe e o ritmo e frequência de sugadas do bebê 23 .

Considerando-se que atualmente a mulher brasileira trabalhadora formal tem o direito a 120 dias de licença maternidade remunerada e, em algumas situações, a ter condições adequadas no ambiente de trabalho, após o seu retorno, para amamentar seus filhos até os seis meses de idade, esperava-se que o maior risco da interrupção do AME estaria entre as mulheres com vínculo informal de trabalho. Contrariamente, verificamos neste estudo que esse tipo de vínculo foi o que associou-se como fator de proteção à interrupção do AME. Nesse caso, a informalidade parece proporcionar maior flexibilidade de horários e, por consequência, maior disponibilidade para lactentes de baixo peso ao nascer, o que por sua vez, pode ter propiciado um aumento nas taxas de AME. Alguns estudos fortalecem esse achado quando apontam que mulheres trabalhadoras formais, mesmo em condições favoráveis, precisam mais do que apenas benefícios trabalhistas para o AME por seis meses 24,25.

De acordo com o Índice Paulista de Vulnerabilidade Social (IPVS) 26, a região estudada concentra famílias cuja combinação de características pode produzir uma deterioração de seu nível de bem-estar, em consequência de sua exposição a determinados tipos de riscos como agravos à saúde, à violência e à pobreza. Além disso, foi notável que a grande maioria dos lactentes identificados como de baixo peso ao nascer era oriunda especificamente da área geográfica considerada de alta vulnerabilidade. Neste estudo foram observados fatores de risco, tais como idade jovem; inserção no mercado de trabalho, provavelmente, com inflexibilidade nos horários; e uso inadequado de bebida alcoólica. Situando-se tais fatores no contexto de vulnerabilidade social registrada na área, é possível visualizar uma potencialização das dificuldades, enfrentadas pelos profissionais de saúde da Atenção Básica, nas intervenções específicas para a redução dos níveis da interrupção precoce do AME.

Entre os aspectos gestacionais, a realização de seis ou mais consultas tem sido uma das reco- 
mendações mais enfatizadas pelas políticas deste âmbito para a redução das taxas de mortalidade materna e melhoria do resultado perinatal 5. O achado do presente estudo, mostrando que as mulheres que realizaram menos de seis consultas no pré-natal, relativamente àquelas com número adequado, tinham maior probabilidade de interromperem o AME até o terceiro mês, concordando com o estudo de bebês de alto risco 18 , reforçam e dão sustentação a esta recomendação, pelo menos no que diz respeito à duração do AME.

Por outro lado, observou-se, também, que o fator "não receber orientação sobre aleitamento materno no pré-natal" não apresentou associação com a interrupção do AME. Isso mostra certa dissonância entre os próprios resultados do presente estudo, pois, se as gestantes que realizaram um número adequado de consultas tiveram mais resultados positivos em relação ao aleitamento, supõe-se que ações educativas inseridas nos atendimentos pré-natais mostrariam também a efetividade esperada. Pela metodologia quantitativa empregada no presente estudo não foi possível levantar nenhuma hipótese explicativa para essa contradição.

No tocante às falhas nas ações educativas no ciclo gravídico puerperal, por investigações de abordagens tanto quantitativas quanto qualitativas, sabe-se que quando fatores subjetivos das mulheres não são levados em conta, a comunicação entre profissionais e pacientes não se estabelece efetivamente e ficam prejudicados tanto o vínculo com o serviço de saúde quanto a aderência às orientações recebidas 13,27,28,29.

Esses achados corroboraram para o reconhecimento de que, isoladamente, o número de consultas de pré-natal não garante a qualidade dos cuidados prestados à gestante, e assim, o apoio emocional e uma abordagem mais acolhedora às gestantes e puérperas têm sido práticas preconizadas pela Rede Amamenta Brasil 11.

Dos fatores relacionados ao nascimento, o peso abaixo ou igual a $2.000 \mathrm{~g}$ mostrou associação com a interrupção do AME até o terceiro mês, comparando-se com o peso superior a este. O baixo peso ao nascer tem sido apontado como fator de risco para a interrupção precoce do AME 18,20. Contudo, note-se que nesses estudos o critério classificatório do baixo peso ao nascer é o de 2.500 g ou menos e a comparação de associação com AME é feita com os bebês acima deste peso. Portanto, pareando-se esses achados aos nossos, pode-se levantar a hipótese da existência de um gradiente dose resposta para a interrupção do AME. Ou seja, à medida que diminui o peso ao nascer aumenta a probabilidade de interromper a amamentação, o que é coerente, uma vez que quanto menor o peso ao nascer maior a gravida- de e, provavelmente, igualmente maior a exposição aos obstáculos iniciais da amamentação.

Resultado não apresentado, porém digno de nota é que, apesar de boa parte da população estudada ter sido atendida em região da capital paulista, cujos hospitais adotavam a Iniciativa Amigo da Criança 30 e o Método Canguru 4, nenhuma diferença nesta população, com relação à duração do AME, foi detectada pelas análises realizadas. Uma possível explicação para esse fato pode estar relacionada às desigualdades no uso de tecnologias previstas pelo Método Canguru nos hospitais, sugeridas nos resultados apresentados na avaliação sobre este Método no Brasil 6. Dessa forma, pode-se aplicar a mesma explicação para os hospitais com o título de Amigo da Criança, sendo que os achados do presente trabalho concordam com os resultados de estudo na Atenção Básica do Rio de Janeiro 15.

Os conhecimentos atuais mostram que as mulheres têm condições de manter a lactação de modo suficiente para gêmeos ou trigêmeos, uma vez que a produção láctea depende do ritmo e das condições adequadas de sucção dos bebês para sua manutenção. Ao contrário, nossos resultados mostraram que mães com partos múltiplos tiveram maior probabilidade de interromper prematuramente o AME, em relação às com partos únicos. A explicação para esse achado parece estar ligada ao fato de se tratar de lactentes de baixo peso ao nascer e prematuros, quando as dificuldades maternas no estabelecimento e manutenção prolongada do AME podem ser mais intensas. Nesse sentido, estudos com gemelares, inclusive os prematuros 31, têm evidenciado maior vulnerabilidade para interrupção da amamentação nessa população, e que a sua superação está relacionada com a existência de um suporte extra ou com os conhecimentos prévios da mãe sobre o tema ${ }^{32}$. Isso sugere que a manutenção do aleitamento sofre influência positiva de um suporte profissional no pré-natal e puerpério.

A dificuldade das mães nas primeiras mamadas (ainda na fase hospitalar), a queixa da mãe sobre a mamada no primeiro mês e o uso da chupeta no primeiro e segundo meses (fase ambulatorial) como fatores que mostraram associação com a interrupção do AME são outros resultados da presente pesquisa que só podem ser compreendidos à luz de conhecimentos resultantes de estudos de delineamentos qualitativos.

Pesquisas sobre as representações maternas acerca da amamentação de lactentes nascidos com baixo peso e prematuros 33 constataram que para a maioria das mulheres a experiência da amamentação é um processo desgastante, permeado de dificuldades, incertezas, ansiedades e culpa. Esses processos subjetivos viven- 
ciados pela mãe no início da vida do bebê de baixo peso, mesmo para aquelas que desejavam muito amamentar, propiciaram a iminência do fracasso do aleitamento materno, face às inúmeras variáveis estressantes a que foram expostas (tanto na vivência hospitalar como na dinâmica familiar) 34 as quais colaboram para diminuir a produção e ejeção do leite 33 . Mesmo para aquelas mães que conseguiram restabelecer sua autoconfiança e obter sucesso na amamentação, podem ser, ainda, consideradas vulneráveis às críticas sociais nos diversos aspectos que englobam o cuidado aos recém-nascidos de baixo peso ou prematuro 34 , desencadeando a introdução precoce de outros alimentos e o uso de bicos artificiais (chupetas e mamadeiras) 33,35.

O uso da chupeta pelos lactentes como fator de risco para a menor duração do AME passou a ser um tema controverso, na medida em que algumas investigações apontam-no como um marcador de dificuldades na amamentação 14 por poder ter sido introduzido como consequência de problemas nesta prática; e outras reafirmamno como fator de risco 22,36, inclusive para os lactentes de baixo peso 7,35, como no nosso caso.

Pelos resultados discutidos pode-se concluir que durante os primeiros meses de desenvolvimento do bebê o sucesso da amamentação defronta-se com inúmeros obstáculos que devem ser adequadamente manejados para a sua superação. Os principais aspectos apontados por outros autores para dirimir eventuais dificuldades das mães nessa prática dizem respeito à qualidade da atenção prestada às mulheres, tanto nas maternidades quanto na Atenção Básica. Especial atenção deve ser dada aos recursos humanos que precisam estar preparados, além de tecnicamente para a promoção do aleitamento materno, também para uma abordagem pautada no aconselhamento 11,12 , com respeito às crenças e à cultura das mulheres com suas diferentes histórias de vida 13,22,27,28. Tudo indica que o uso das habilidades de ouvir e acolher dos profissionais de saúde e a sua capacidade de estabelecer confiança e de dar apoio às mães aumentam a prática do AME e retarda a introdução da mamadeira, leite artificial, bem como da chupeta, inclusive entre os lactentes de baixo peso 1,7,17,19,35.

Nesse sentido, tanto o uso da chupeta quanto a presença prolongada de queixa das mães no seguimento ambulatorial devem ser considerados como um alerta aos profissionais de saúde para a prática do AME, requerendo uma abordagem mais singular.

No tocante às limitações do estudo, não se pode deixar de mencionar o viés de memória. Com relação às orientações recebidas no prénatal, as mães que apresentaram mais dificul- dades na amamentação durante o período prolongado de internação para os cuidados do bebê e também durante a fase ambulatorial podem ter se recordado mais das orientações recebidas, generalizando-as também para o período do prénatal. Portanto, mais mães que responderam ter recebido orientações no pré-natal podem ter interrompido mais precocemente o AME, contribuindo para minimizar a força de associação deste fator com o desfecho de interesse.

No que diz respeito ao viés de seleção, não se pode descartá-lo, porém acredita-se que ao convidar a participar do estudo todas as mães que preencheram os critérios de inclusão e a semelhança observada entre participantes e não participantes segundo diversas características dos lactentes e das mães (idade, escolaridade, trabalho, idade gestacional, tipo de gestação, pré-natal e peso ao nascer) essa possibilidade seja mínima. De qualquer forma, acredita-se que os resultados aqui apresentados são representativos daquilo que ocorre nas UBS estudadas e sua generalização deve ser feita com cautela.

A discussão aqui exposta demonstra a complexidade do processo que determina a continuidade ou não do AME nessa população. Além disso, constatou-se também que a população ora em questão, moradores em áreas periféricas de uma grande metrópole, está exposta a riscos adicionais de outras naturezas, desde questões relacionadas à violência até a problemas de acesso aos serviços de saúde, o que nos leva a afirmar que a discussão deste tema não se esgota neste estudo.

Ressaltamos alguns aspectos que fortalecem os resultados aqui analisados. Um deles se refere ao rigor na coleta de dados e, portanto, à qualidade das informações do estudo, haja vista o esforço constante na articulação entre pesquisadores, gestores e profissionais de saúde das UBS. Some-se a isso um trabalho minucioso dos pesquisadores em supervisão semanal do campo para completar e checar os dados por meio de contato direto com as mães participantes do estudo. Um aspecto inovador foi o fato de se ter investigado os fatores de risco para a interrupção precoce da prática do AME em contexto do seguimento ambulatorial na Atenção Básica, uma vez que na maioria dos trabalhos o foco é o período hospitalar.

O conhecimento prévio dos fatores associados à interrupção do AME de lactentes nascidos com baixo peso pode facilitar o planejamento de ações e políticas locais no sentido de melhorar os índices de aleitamento materno, visando a reduzir a morbimortalidade infantil, uma vez que a maioria dos fatores identificados neste estudo é passível de intervenção ao longo do seguimento ambulatorial. 


\section{Resumo}

Visou-se a identificar os fatores associados à interrupção do aleitamento materno exclusivo (AME) de lactentes nascidos com baixo peso assistidos na Atenção Básica. Estudo transversal com 170 lactentes assistidos em unidades básicas de saúde (UBS) da periferia do Município de São Paulo, Brasil. Incluíram-se nascidos com peso $\leq 2.500 \mathrm{~g}$ (inclusive gemelares), Apgar (5') $\geq 7$ e acompanhados até o terceiro mês. Excluíram-se bebê/mãe com alterações que impedissem o AME e óbitos. Coletaram-se os dados utilizando-se formulários preenchidos nas consultas e prontuários. As razões de prevalência foram obtidas por regressão de Poisson. Identificou-se associado à interrupção do AME no terceiro mês: idade materna $<18$ anos; vínculo empregatício informal (como fator de proteção); ingestão de álcool na gestação; < 6 consultas no pré-natal; gestação múltipla; peso ao nascer $\leq 2.000$; dificuldade na primeira mamada; queixa sobre a amamentação no primeiro mês; uso de chupeta no primeiro e segundo meses. O conhecimento desses fatores beneficia o planejamento de ações e políticas locais visando ao aumento da amamentação exclusiva dos lactentes nascidos com baixo peso.

Recém-Nascido de Baixo Peso; Aleitamento Materno; Atenção Primária à Saúde

\section{Referências}

1. Aguayo J. Maternal lactation for preterm newborn infants. Early Hum Dev 2001; 65 Suppl:S19-29.

2. Nascimento MBR, Issler H. Aleitamento materno em prematuros: manejo clínico hospitalar. J Pediatr (Rio J.) 2004; 80(5 Suppl):S163-72.

3. Venâncio SI, Almeida H. Método Mãe Canguru: aplicação no Brasil, evidências científicas e impacto sobre o aleitamento materno. J Pediatr (Rio J.) 2004; 80(5 Suppl):S173-80.

4. Ministério da Saúde. Atenção humanizada ao recém-nascido de baixo peso: Método Canguru. http://portal.saude.gov.br/portal/arquivos/pdf/ manual_canguru.pdf (acessado em 05/Jan/2011).

5. Ministério da Saúde. Pré-natal e puerpério atenção qualificada e humanizada. http://portal.saude.gov. br/portal/arquivos/pdf/manual_puerperio_2006. pdf (acessado em 05/Jan/2001).

6. Lamy Filho F, Silva AAM, Lamy ZC, Gomes MASM, Moreira MEL; Grupo de Avaliação do Método Canguru. Avaliação dos resultados neonatais do Método Canguru no Brasil. J Pediatr (Rio J.) 2008; 84:428-35.

\section{Colaboradores}

M. T. C. Sanches coordenou o estudo e participou em todas as etapas da produção do artigo. G. S. Buccini contribuiu na execução da pesquisa (coleta de dados, supervisão de campo e das informações coletadas), análise dos resultados, confecção das tabelas e participação na redação do texto. S. G. A. Gimeno contribuiu para análise dos dados, participação na elaboração e discussão dos resultados e redação do texto. T. E. C. Rosa contribuiu na elaboração do estudo, organização dos resultados, discussão dos mesmos e redação do texto. A. W. Bonamigo contribuiu na execução do estudo, participação na discussão dos resultados e redação do texto.

\section{Agradecimentos}

Agradecemos à Coordenação de Saúde da Supervisão Técnica de Saúde de Pirituba/Perus da Secretaria de Saúde do Município de São Paulo pelo apoio prestado por todas as equipes de saúde das UBS que participaram deste estudo. Agradecemos ainda o apoio financeiro recebido pela Fundação de Amparo à Pesquisa do Estado de São Paulo (FAPESP, processo 2005/60654-8).

7. Sanches MTC. Estudo dos fatores associados à amamentação exclusiva de recém-nascidos de baixo peso ao nascer, integrantes do Método Mãe Canguru [Tese de Doutorado]. São Paulo: Faculdade de Saúde Pública, Universidade de São Paulo; 2005.

8. Almeida H, Venâncio SI, Sanches MTC. Impacto do Método Canguru nas taxas de aleitamento materno exclusivo em recém-nascidos de baixo peso. J Pediatr (Rio J.) 2010; 86:250-3.

9. Penalva O, Schwartzman JS. Estudo descritivo do perfil clínico-nutricional e do seguimento ambulatorial de recém-nascidos prematuros atendidos no Programa Método Mãe Canguru. J Pediatr (Rio J.) 2006; 82:33-9.

10. Ramanathan K, Paul VK, Deorari AK, Taneja U, George G. Kangaroo mother care in very low birth weight infants. Indian J Pediatr 2001; 68:1019-23.

11. Ministério da Saúde. Rede Amamenta Brasil: caderno do tutor. http://bvsms.saude.gov.br/bvs/ publicacoes/rede_amamenta_brasil_caderno_tu tor.pdf (acessado em 05/Jan/2011). 
12. Bueno LGS, Teruya KM. Aconselhamento em amamentação e sua prática. J Pediatr (Rio J.) 2004; 80(5 Suppl):S126-30.

13. De Oliveira MIC, Camacho LAB. Impacto das Unidades Básicas de Saúde na duração do aleitamento materno exclusivo. Rev Bras Epidemiol 2005; 5:41-51.

14. Victora CG, Behague DP, Barros FC, Olinto MT, Weiderpass E. Pacifier use and short breastfeeding duration: cause, consequence or coincidence? Pediatrics 1997; 99:445-53.

15. Pereira RSV, De Oliveira MIC, Andrade CLT, Brito AS. Fatores associados ao aleitamento materno exclusivo: o papel do cuidado na atenção básica. Cad Saúde Pública 2010; 26:2343-54.

16. Vannuchi MTO, Monteiro CA, Réa MF, Andrade SM, Matsuo T. Iniciativa Hospital Amigo da Criança e aleitamento materno em unidade de neonatologia. Rev Saúde Pública 2004; 38:422-8.

17. Agrasada GV, Gustafsson J, Kylberg E, Ewald U. Postnatal peer counseling on exclusive breastfeeding of low-birthweight infants: a randomized controlled trial. Acta Paediatr 2005; 94:1109-15.

18. Mancini PGB, Meléndez GV. Aleitamento materno exclusivo na alta de recém-nascidos internados em berçário de alto risco e os fatores associados a essa prática. J Pediatr (Rio J.) 2004; 80:241-8.

19. Leite AJM, Puccini RF, Atalah AN, Cunha ALA, Machado MMT. Effectiveness of home-based peer counseling to promote breastfeeding in the northeast of Brazil: a randomized clinical trial. Acta Paediatr 2005; 94:741-6.

20. Venâncio SI, Monteiro CA. Individual and contextual determinants of exclusive breast-feeding in São Paulo, Brazil: a multilevel analysis. Public Health Nutr 2006; 9:40-6.

21. World Health Organization. World Health Organization's infant feeding recommendation. Indicators for assessing breastfeeding Practices. Geneva: World Health Organization; 1991.

22. Chaves RG, Lamounier JA, César CC. Fatores associados com a duração do aleitamento materno. J Pediatr (Rio J.) 2007; 83:241-6.

23. Kachani AT, Okuda LS, Barbosa ALR, Brasiliano S, Hochgraf PB. Aleitamento materno: quanto o álcool pode influenciar na saúde do bebê? Pediatria (São Paulo) 2008; 30:249-56.

24. Réa MF, Venâncio SI, Batista LE, Santos RG, Greiner T. Possibilidades e limitações da amamentação entre trabalhadoras formais. Rev Saúde Pública 1997; 31:149-56.
25. Osis MJD, Duarte GA, Pádua KS, Hardy E, Sandoval $\mathrm{M}$, Bento SF. Aleitamento materno exclusivo entre trabalhadoras com creche no local de trabalho. Rev Saúde Pública 2004; 38:172-9.

26. Fundação Sistemas Estadual de Análise de Dados. Índice Paulista de Vulnerabilidade Social. http:// www.seade.gov.br/produtos/ipvs/analises (acessado em 01/Jun/2008).

27. Almeida ICAL, Tanaka OY. Perspectiva das mulheres na avaliação do Programa de Humanização do Pré-natal e Nascimento. Rev Saúde Pública 2009; 43:98-104.

28. Ramos CV, Almeida JAG. Aleitamento materno: como é vivenciado por mulheres assistidas em uma unidade de saúde de referência na atenção materno-infantil em Teresina, Piauí. Rev Bras Saúde Matern Infant 2003; 3:315-21.

29. Oliveira MIC, Camacho LAB, Tedstone AE. Extending breastfeeding duration through primary care: a systematic review of prenatal and postnatal interventions. J Hum Lact 2001; 17:326-43.

30. World Health Organization/United Nations Children's Fund. The global criteria for the Baby Friendly Hospital Initiative. Geneva: World Health Organization/New York: United Nations Children's Fund; 1992.

31. Ostlund A, Nordstrom M, Dykes F, Flacking R. Breastfeeding in preterm and term twins-maternal factors associated with early cessation: a population-based study. J Hum Lact 2010; 26:235-41.

32. Rozas MR, Costa J, Cambredó MV, Colomé N, Bravo MT, Grau M. Lactancia materna y parto gemelar. Aten Primaria 2000; 26:224-30.

33. Javorski M. As representações sociais do aleitamento materno para mães de prematuros em unidade de cuidado canguru. Rev Latinoam Enferm 2004; 12:890-8.

34. Caetano LC, Scochi CGS, Angelo M. Vivendo no método canguru a tríade mãe-filho-família. Rev Latinoam Enferm 2005; 13:562-8.

35. Cunha AJLA, Leite AM, Machado MMT. Breastfeeding and pacifier use in Brazil. Indian J Pediatr 2005; 72:13-6.

36. Parizoto GM, Parada CMGL, Venâncio S, Carvalhaes MABL. Tendência e determinantes do aleitamento materno exclusivo em crianças menores de 6 meses. J Pediatr (Rio J.) 2009; 85:201-8.

Recebido em 01/Out/2010

Versão final reapresentada em 18/Fev/2011

Aprovado em 28/Fev/2011 